\title{
Oral administration of a galactooligosaccharide preparation inhibits development of atopic dermatitis-like skin lesions in NC/Nga mice
}

\author{
SOICHI TANABE and SATOMI HOCHI \\ Graduate School of Biosphere Science, Hiroshima University, 1-4-4 Kagamiyama, \\ Higashi-Hiroshima 739-8528, Japan
}

Received September 25, 2009; Accepted November 2, 2009

DOI: 10.3892/ijmm_00000349

\begin{abstract}
Anti-allergic effects of galactooligosaccharide (GOS), which is found in breast milk and frequently added to food for promoting health, were evaluated in a human-like mouse model of atopic dermatitis (AD). NC/Nga mice were fed $5.5 \%$ GOS for 8 weeks, and we examined whether this treatment suppressed the development of AD-like skin lesions in these mice. Mice fed GOS exhibited significantly less symptoms of dermatitis, reduced scratching frequency, and lower levels of serum total immunoglobulin E compared to control. At the end of the 8-week-experimental period, spleens were removed, and the splenocytes were stimulated with phorbol 12-myristate 13-acetate and ionomycin, following which production of cytokines and a chemokine was analyzed. Elevated levels of Th1 cytokines such as interferon- $\gamma$ were observed in splenocytes from GOS-fed mice. However, the levels of Th2 cytokines such as interleukin (IL)-13 were unchanged. Furthermore, GOS inhibited the production of inflammatory cytokines such as IL-1ß, IL-6, IL-17, and tumor necrosis factor- $\alpha$ but enhanced production of immunomodulatory IL-10. The results indicate that GOS effectively blocked AD-like skin lesions in the mice by at least partly inducing production of IL-10 and suppressing the production of cytokines such as IL-17, which are involved in skin inflammation.
\end{abstract}

\section{Introduction}

More than $25 \%$ of the population in industrialized countries suffers from immunoglobulin (IgE)-mediated (type I) allergic symptoms (1). The disease arises when mechanisms controlling responses to innocuous environmental allergens

Correspondence to: Dr Soichi Tanabe, Graduate School of Biosphere Science, Hiroshima University, 1-4-4 Kagamiyama, Higashi-Hiroshima 739-8528, Japan

E-mail: stanabe@hiroshima-u.ac.jp

Key words: galactooligosaccharide, $\mathrm{NC} / \mathrm{Nga}$, skin lesion, IgE, cytokine, Th17, IL-17 break down. Although the reasons why certain individuals experience particular types of hypersensitivity are unclear, there is evidence that both genetic and environmental factors influence susceptibility (2). Analyses of genes contributing to allergic disorders have shown that susceptibility arises from complex multigenic interactions (3). The recent dramatic increase in prevalence of allergic sensitization provides evidence for the additional roles of environmental factors in the pathogenesis of immune hypersensitivity.

An increasing number of studies have suggested an important role of intestinal microflora in prevention of allergic diseases. For example, differences in the intestinal microbiota between allergic and non-allergic children were demonstrated; allergic children were less often colonized with lactobacilli and bifidobacteria and harbored a higher number of aerobic microorganisms compared to non-allergic children $(4,5)$. Thus, manipulating the intestinal microflora with probiotics and/or prebiotics is a possible way of preventing or treating atopic dermatitis (AD) (6). A Finnish study was the first to report that the frequency of $\mathrm{AD}$ in neonates treated with Lactobacillus rhamnosus GG was almost half that in those receiving placebo (7). However, there is little agreement about the inhibitory effect of probiotics on development of AD (8).

Prebiotics, which are nondigestible food components that benefit the host by selectively stimulating the growth or activity of nonpathogenic bacteria in the colon, are also candidates for preventing/treating allergic disorders such as AD. For example, the effect of a mixture of 4 probiotic bacterial strains plus prebiotic galactooligosaccharide (GOS) in preventing allergic diseases was evaluated in pregnant women and their infants (9).

GOS is generally manufactured from lactose using the enzyme $\beta$-galactosidase to attach glycosyl residues, which results in complex mixtures with various glycosidic linkages (10). Oligomate (Yakult Pharmaceutical Industry, Tokyo, Japan), the commercially available GOS preparation used in the present study, is composed of 55\% Gal-(Gal)n-Glc ( $n=1-3)$ with B1-4 linkage and Gal-Glc (ß1-3) and 45\% lactose and monosaccharides. The main component is 4 '-galactocyl lactose [Gal-Gal-Glc (ß1-4)], which is found in breast milk. GOS stimulates the growth and activity of bifidobacteria and lactobacilli in the colon. In Japan, GOS is approved by the Ministry of Health, Labor, and Welfare as a Food for Specified 
A

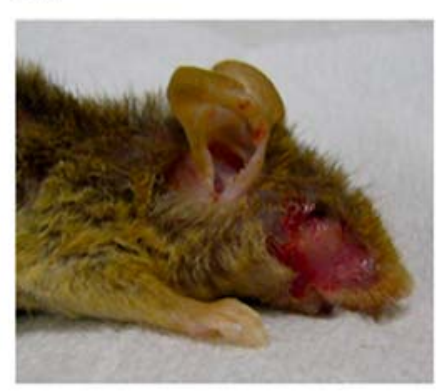

Severe
B

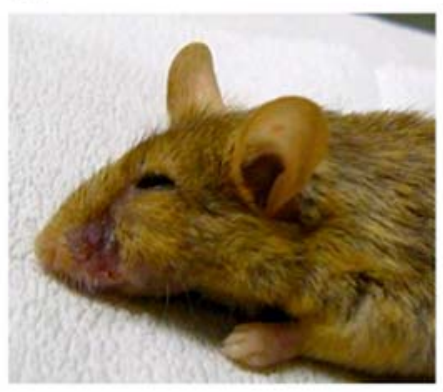

Mild

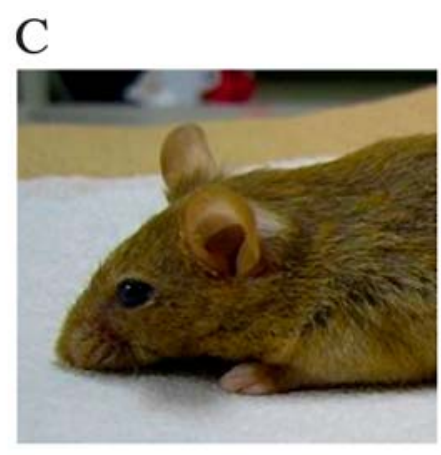

GOS-fed

Control

Figure 1. Inhibitory effect of GOS on skin inflammation in NC/Nga mice. Representative photographs of 12-week-old mice are shown. (A and B) Control mice showed severe (A) or mild (B) inflammation. (C) GOS-fed mice.

Health Uses (FOSHU), for consumption to maintain/promote health or for special health uses by people wishing to control their health conditions. In case of GOS, a food including $>5 \mathrm{~g}$ /day of GOS is currently approved its ability with modifying gastrointestinal conditions and is given a chance to make such a health claim.

In this study, the anti-allergic effects of GOS were evaluated in a human-like model of $\mathrm{AD}, \mathrm{NC} / \mathrm{Nga}$ mice. The mice were fed $5.5 \%$ GOS for 8 weeks, and scratching behavior and clinical symptoms were evaluated weekly. After the experimental period, serum total $\mathrm{IgE}$ and cytokine production by splenocytes were evaluated.

\section{Materials and methods}

Reagents. RPMI-1640 medium, penicillin, and streptomycin were purchased from Invitrogen (Carlsbad, CA). Fetal bovine serum (FBS) was obtained from ICN Biomedicals (Osaka, Japan). Phorbol 12-myristate 13-acetate (PMA) and ionomycin were purchased from Wako Pure Industries (Osaka, Japan) and EMD Bioscience (Darmstadt, Canada), respectively. All other chemicals were of reagent grade.

Animals and diets. Four-week-old female NC/Nga mice were purchased from Japan SLC (Shizuoka, Japan) and housed in a room with controlled temperature $\left(24^{\circ} \mathrm{C}\right)$ and a $12 \mathrm{~h}$ light/ $12 \mathrm{~h}$ dark cycle under conventional conditions. The mice were given free access to food and water and maintained according to Hiroshima University's Guide for the Care and Use of Laboratory Animals. They were divided into 2 groups ( $n=6$ each); one group (control) was fed the AIN-93G diet and another the AIN-93G diet in which a GOS preparation was substituted for sucrose (Table I). The GOS preparation used in the study (Oligomate 55NP) contained 55\% GOS and $45 \%$ monosaccharide and lactose, and was obtained from Yakult Pharmaceutical Industry. Both the AIN-93G and GOScontaining diet were prepared by Oriental Yeast (Tokyo, Japan). The mice were maintained on the diets for 8 weeks during which scratching behavior and clinical symptoms were evaluated weekly according to the method of Onishi et al
Table I. Composition of diets of control and GOS-fed mice.

\begin{tabular}{lcc}
\hline Ingredient $(\mathrm{g} / \mathrm{kg})$ & $\begin{array}{c}\text { Control diet } \\
(\text { AIN93G) }\end{array}$ & $\begin{array}{c}\text { Diet containing } \\
5.5 \% \mathrm{GOS}\end{array}$ \\
\hline Cornstarch & 397.5 & 397.5 \\
Casein & 200 & 200 \\
$\alpha$-cornstarch & 132 & 132 \\
Sucrose & 100 & 0 \\
Oligomate 55NPa & 0 & 100 \\
Soybean oil & 70 & 70 \\
Cellulose & 50 & 50 \\
AIN93G mineral & 35 & 35 \\
AIN93G vitamin & 10 & 10 \\
L-cystine & 3 & 3 \\
Choline bitartrate & 2.5 & 2.5 \\
$t$-butylhydroquinone & 0.014 & 0.014 \\
\hline
\end{tabular}

${ }^{a}$ Oligomate 55NP contains 55\% GOS.

(11) with some modifications. At 12 weeks of age, serum total IgE titers were determined following which the mice were sacrificed and cytokine and chemokine production by splenocytes was evaluated.

Evaluation of the severity of dermatitis. According to the method of Matsuda et al (12), the severity of dermatitis was assessed once a week by assigning a score for each of the 5 symptom items (scratching behavior, hemorrhage, edema, excoriation/erosion, and xerosis/dryness). The scale used was 0 (no symptoms), 1 (mild), 2 (moderate), and 3 (severe) and the total score was expressed as the sum of the scores of the 5 items (total score, 15). For scratching behavior, the number of scratching incidents per 3 min was assessed. 

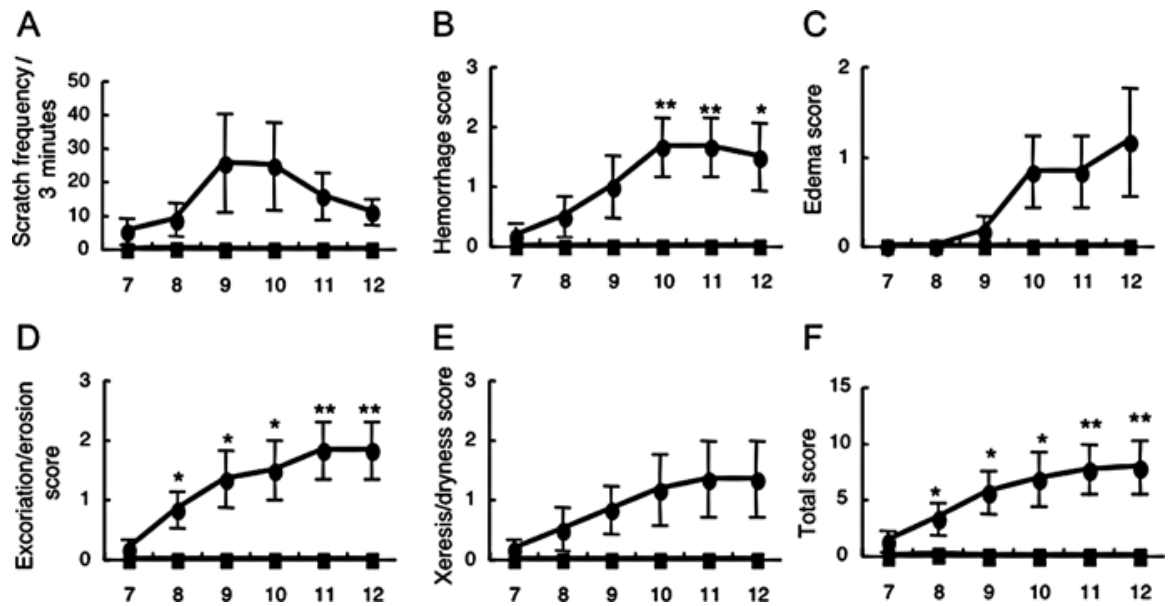

Figure 2. Effect of GOS on clinical scores of skin symptoms. (A) Numbers of scratching incidents per 3 min. (B-E) The severity of dermatitis was assessed by scoring from 0 (no symptoms), 1 (mild), 2 (moderate), and 3 (severe) for hemorrhage (B), edema (C), excoriation/erosion (D), and xerosis/dryness (E). Severity of dermatitis was expressed as the sum of the respective scores obtained for the 5 symptoms (F). $\bullet$, control mice; $\mathbf{m}$, GOS-fed mice. $\mathrm{n}=6$ for each group. ${ }^{*} \mathrm{p}<0.05,{ }^{* *} \mathrm{p}<0.01$.

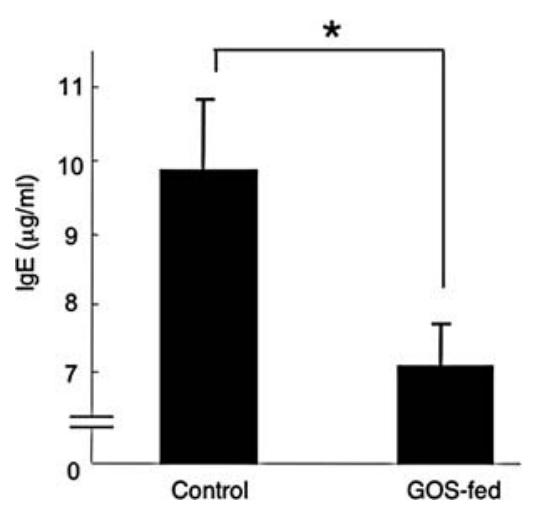

Figure 3. Inhibitory effect of GOS on total IgE levels in NC/Nga mice. $n=6$ for each group. ${ }^{*} \mathrm{p}<0.05$.

These numbers were then converted to scores 0-3 for summation of the 5 items.

Measurement of serum total IgE. After the 8-weekexperimental period, blood was collected from a tail vein. Serum samples were obtained by centrifugation and stored at $-80^{\circ} \mathrm{C}$ until use. Total serum IgE levels were measured using a sandwich ELISA kit (Bethyl laboratories, Inc., Montgomery, TX) according to the manufacturer's instructions.

Measurement of cytokines and chemokine. At the end of the 8-week-experimental period, mice were sacrificed by cervical dislocation and the spleen was removed. Based on the skin lesion scores, the control mice were further divided into 2 groups, 1 with severe and 1 with mild lesions ( $\mathrm{n}=3$ each). Splenocytes from the 3 mice in severe and mild lesion groups were pooled, and splenocytes from all 6 mice of the GOS-fed group were pooled. All animal studies were conducted according to the University ethics guidelines on animal care and experimentation.

The splenocytes $\left(2 \times 10^{7}\right.$ cells $)$ were incubated with PMA $(25 \mathrm{ng} / \mathrm{ml})$ and ionomycin $(1 \mu \mathrm{g} / \mathrm{ml})$ in $1 \mathrm{ml}$ of PRMI-1640 medium with $10 \%$ FBS, $0.05 \mathrm{mM}$ 2-mercaptoethanol, $0.01 \mathrm{M}$ 2- [4-(2-hydroxyethyl)-1-piperazinyl] ethanesulfonic acid (HEPES), penicillin, and streptomycin. Cells were cultured at $37^{\circ} \mathrm{C}$ under a humidified $5 \% \mathrm{CO}_{2}$ atmosphere for $72 \mathrm{~h}$, and supernatants were harvested for determining cytokine and chemokine concentrations by the microbead method using the Bio-Plex Suspension Array System (BioRad Laboratories, Hercules, CA, USA), as described previously (13).

Briefly, culture supernatants were incubated with beads conjugated with anti-granulocyte-colony stimulating factor (G-CSF), anti-interleukin (IL)-1ß, -IL-2, -IL-4, -IL-6, -IL-10, -IL-13, -IL-17, anti-interferon (IFN)- $\gamma$, anti-tumor necrosis factor (TNF)- $\alpha$, and anti-monocyte chemoattractant (chemotactic) protein (MCP)-1 antibodies followed by a sandwich immunoassay using biotinylated secondary antibodies. The beads were washed three times during each incubation. Phycoerythrin-streptavidin was used as a reporter. The relative fluorescence units were detected by counting 100 beads with the Bio-Plex apparatus. Data were evaluated with the Bio-Plex Manager Software 3.0 (Bio-Rad Laboratories) using 5PL curve fitting.

Statistical analysis. Results were expressed as mean \pm SE. Statistical analysis was performed using Student's t-test.

\section{Results}

GOS inhibited development of skin lesions accompanied by lower serum IgE. NC/Nga mice were previously shown to spontaneously develop AD-like skin lesions under conventional conditions. In the present study, 4-week-old NC/ Nga mice were maintained for 8 weeks (from 4 to 12 weeks of age) on a diet with or without GOS. Dermatitis was prominent from 8 to 9 weeks of age in the control mice. At 12 weeks of age, approximately half of the mice in the control group manifested severe dermatitis, particularly around the eyes and ears (Fig. 1A) and the remaining half showed mild inflammation (Fig. 1B). However, no AD-like skin lesions were observed in the GOS-fed mice (Fig. 1C). 
Table II. Effects of GOS on cytokine and chemokine production by mouse splenocytes $(\mathrm{pg} / \mathrm{ml})$.

\begin{tabular}{lccc}
\hline & \multicolumn{2}{c}{ Control $^{\mathrm{a}}$} & \\
\cline { 2 - 3 } Group & Severe & Mild & GOS-fed \\
\hline Th1 cytokines & & & \\
IFN- $\gamma$ & 69 & 196 & 238 \\
$\quad$ IL-2 & 64 & 270 & 487 \\
Th2 cytokines & & & \\
IL-4 & 10 & 26 & 34 \\
$\quad$ IL-13 & 60 & 72 & 60 \\
Treg cytokine & & & \\
$\quad$ IL-10 & 56 & 150 & 165 \\
Th17 cytokine & & & \\
$\quad$ IL-17 & 1,507 & 1,351 & 874 \\
Inflammatory cytokines & & & \\
and chemokine & & & \\
IL-1ß & 39 & 21 & 16 \\
IL-6 & 1,604 & 1,663 & 1,024 \\
$\quad$ G-CSF & 71 & 32 & 41 \\
MCP-1 & 7,435 & 6,258 & 4,076 \\
$\quad$ TNF- $\alpha$ & 26 & 14 & 8 \\
\hline
\end{tabular}

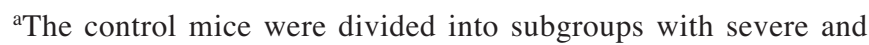
mild lesions ( $\mathrm{n}=3$ for each subgroup).

As shown in Fig. 2A, an increase in scratching behavior was evident from 9 weeks of age in the control mice, but this symptom was inhibited in the GOS-fed mice. The mice in the control group gradually developed AD-like lesions (Fig. 2B-E). Two mice in the control group had a score of 3 (severe) for all 5 score items. In contrast, the GOS-fed mice completely inhibited development of AD-like lesions, and significant differences in the severity scores were observed between the control and GOS-fed mice from 8 weeks of age (Fig. 2F). The serum total IgE level of the GOS-fed group was significantly lower than that of the control group at 12 weeks of age (Fig. 3).

GOS increases Th1 cytokine production. To examine the anti-allergic effects of GOS in NC/Nga mice, we investigated cytokine production by splenocytes. Based on the results of skin lesions, the control mice were further divided into groups with severe and mild lesions. As shown in Table II, the production of Th1 cytokines (IFN- $\gamma$ and IL-2) in splenocytes was elevated in the GOS-fed mice compared to the control mice. In concert with the severity of skin lesion, these cytokine levels were lowered. The concentrations of Th2 cytokines (IL-4 and IL-13) were relatively low. In particular, the IL-4 level in the severe control group was very low. No marked differences in the concentration of IL-13 was observed between the 3 groups of mice.

GOS improved Treg/Th17 balance and exerted antiinflammatory activity. To examine the anti-inflammatory effects of GOS in NC/Nga mice, we focused on the Treg/
Th17 balance. As shown in Table II, the level of the inflammatory Th17 cytokine (IL-17) was much lower in the GOS-fed mice compared to the severe and mild control groups. Similarly, the levels of the inflammatory cytokines IL-1 $13, \mathrm{~L}-6, \mathrm{G}-\mathrm{CSF}$, and TNF- $\alpha$ and the inflammatory chemokine MCP-1 were also lower in the GOS-fed mice than in the controls. On the other hand, it is noteworthy that the level of Treg cytokine (IL-10) in the GOS-fed group was higher than that in the severe control group (Table II). Therefore, we suggest that the induction of immunoregulatory IL-10 by GOS was at least partly significant in the amelioration of skin inflammation in $\mathrm{NC} / \mathrm{Nga}$ mice.

\section{Discussion}

Herein, we demonstrated that GOS administration alleviated spontaneous AD in NC/Nga mice (Figs. 1 and 2). These mice are widely used for evaluating the anti-allergenic activity of food components. When kept under conventional conditions, $\mathrm{NC} / \mathrm{Nga}$ mice have been reported to spontaneously develop dermatitis with IgE hyperproduction, which is very similar to human AD (11). Furthermore, it has been reported that these mice develop AD-like skin lesions under specific pathogenfree conditions after repeated application of haptens such as picryl chloride $(14,15)$. Both the models are frequently used as a model of human $\mathrm{AD}$. We adopted the spontaneous $\mathrm{AD}$ model in this study.

The mechanisms of the anti-allergic effects of GOS might involve reduction in serum total $\mathrm{IgE}$ (Fig. 3) and modulation of cytokine production (Table II). Clinically, most patients with $\mathrm{AD}$ showed an increased serum IgE level (16), and there is a correlation between serum IgE level and AD severity. Therefore, it was suggested that GOS exerts anti-allergic effects in part by reducing IgE production. The elevated IgE response reflects increased expression of Th2 cytokines. In general, Th2 cells produce antibody-mediated responses, particularly those that are involved in allergy dominated by the IgE isotype. Furthermore, cytokines produced by Th1 cells negatively regulate the function of Th2 cells (and vice versa). In this context, modulating the Th1/Th2 balance is considered important for the suppression of allergy. In the present study, no marked change in the production of IL-13 was observed after GOS administration. However, this is the first study to show that $\beta$-linked GOS elevated IFN- $\gamma$ production and suppressed serum total IgE. In the case of other nondigestible oligosaccharides, there is increasing evidence that raffinose, $\alpha$-linked GOS (17), fructooligo-saccharide (18) and melibiose (19) suppress the allergic reaction in vivo.

In addition, a subset of IL-17-inducing Th cells (Th17) was recently identified $(20,21)$ and shown to play an important role in tissue inflammation. The effects of Treg/Th17 balance on allergic diseases have been extensively studied. Moreover, Koga et al (22) suggested that Th17 cells might aggravate atopic eczema and that Th17 cells may play a pathogenic role in AD. To clarify this point, it is necessary to examine IL-17+ $\mathrm{T}$ cell infiltration into skin lesions of AD.

GOS successfully inhibited IL-17 production by splenocytes of NC/Nga mice in the present study, which was related to the decreased production of the inflammatory cytokines IL-1ß, IL-6, and TNF- $\alpha$ (Table II). In healthy 
elderly volunteers, production of the anti-inflammatory cytokine IL-10 and significant reductions in the production of inflammatory cytokines (IL-6, IL-1ß, and TNF- $\alpha$ ) were also observed in a human trial of a prebiotic trans-GOS mixture (B-GOS) (23). In addition, we clarified that the levels of G-CSF and MCP-1 were also lowered by GOS. Since it has recently been reported that G-CSF and MCP-1 may be crucially involved in the IL-17A-induced inflammation (24), it is strongly concluded that GOS suppresses IL-17-related inflammation in NC/Nga mice.

Our group has recently focused on the inhibition of IL-17/ Th17 by food components. For example, bifidobacteria suppress IL-17 production in mouse splenocytes and inflamed colon culture (13). We also found that fermented barley extract, prepared from barley shochu residue, suppresses IL-17 production in splenocytes and alleviates AD-like skin lesions in NC/Nga mice (14). Since effective management should be directed not only toward the rapid and adequate relief of inflammation by medicines but also toward sustained improvement in activities of daily living, there may be an increasing requirement for foods with IL-17/Th17-inhibiting activity. Furthermore, Wu et al (25) reported that a commensal bacterium promotes colon cancer by activation of Th17 responses in an animal model. Therefore, GOS may suppress some types of colon tumorigenesis by inhibiting the function of Th17 cells.

Besides, improvement in the intestinal microflora, i.e., a prebiotic effect, would at least partly contribute to the beneficial effects of GOS. Therefore, it is necessary to clarify any changes in the intestinal microflora after GOS administration. In a randomized, double-blind, placebo-controlled trial, GOS was evaluated with concurrent administration of 4 probiotics, Lactobacillus rhamnosus GG, L. rhamnosus LC705, Bifidobacterium breve Bb99, and Propionibacterium freudenreichii ssp. shermanii JS (9). Pregnant women carrying high-risk (for allergy) children were given the probiotic preparation for 2-4 weeks before delivery, and their infants received the same probiotics plus GOS for 6 months. During treatment and at 2 years of age, the cumulative incidence of allergic diseases (food allergy, eczema, asthma, and allergic rhinitis), IgE sensitization, and fecal bacteria were analyzed. The probiotic plus GOS treatment helped to significantly prevent atopic eczema, and the gut of supplemented infants showed more frequently colonized lactobacilli and bifidobacteria. These results suggest an inverse association between atopic diseases and colonization of the gut by probiotics. However, the effect of sole administration of GOS in infants was not elucidated.

Probiotics and prebiotics are also expected to induce secretion of mucosal $\operatorname{Ig} \mathrm{A}$, which participates in antigen elimination and reduction in IgE-associated allergic diseases (26). In this study, feces were collected from each mouse in the last $24 \mathrm{~h}$ of the experiment. Fecal IgA concentrations in the GOS-fed mice did not change significantly but tended to be higher than those in the control mice (data not shown). However, in Balb/c mice, fecal IgA was reported to significantly increase 2 weeks after the start of GOS administration (27). Further studies are necessary to determine whether AD suppression was associated with modulation and/or acceleration of the immune response in the gut.
In conclusion, this study is the first to show that GOS alleviated AD-like skin lesions in NC/Nga mice and improved the Treg/Th17 balance. Considering these results together with the fact that GOS is recognized as a safe prebiotics and is already in use in various parts of the world, we suggest that GOS may be useful in prevention and treatment of AD.

\section{Acknowledgements}

We thank Dr Seiji Kawamoto and Dr Masayuki Shimada (Hiroshima University) for technical suggestions and support. This study was partially supported by ICREO Co. (Tokyo, Japan).

\section{References}

1. Valenta R: The future of antigen-specific immunotherapy of allergy. Nat Rev Immunol 2: 446-453, 2002.

2. Larché M and Wraith DC: Peptide-based therapeutic vaccines for allergic and autoimmune diseases. Nat Med 11: S69-S76, 2005.

3. Cookson W: Genetics and genomics of asthma and allergic diseases. Immunol Rev 190: 195-206, 2002.

4. Björkstén B, Naaber P, Sepp E, et al: The intestinal microflora in allergic Estonian and Swedish 2-year-old children. Clin Exp Allergy 29: 342-346, 1999.

5. Kalliomäki $\mathrm{M}$ and Isolauri E: Role of intestinal flora in the development of allergy. Curr Opin Allergy Clin Immunol 3: 15-20, 2003.

6. van der Aa LB, Heymans HS, van Aalderen WM, et al: Probiotics and prebiotics in atopic dermatitis: review of the theoretical background and clinical evidence. Pediatr Allergy Immunol (In press).

7. Kalliomäki M, Salminen S, Poussa T, et al: Probiotics and prevention of atopic disease: 4-year follow-up of a randomised placebo-controlled trial. Lancet 361: 1869-1871, 2003.

8. Kopp MV and Salfeld P: Probiotics and prevention of allergic disease. Curr Opin Clin Nutr Metab Care 12: 298-303, 2009.

9. Kukkonen K, Savilahti E, Haahtela T, et al: Probiotics and prebiotic galacto-oligosaccharides in the prevention of allergic diseases: a randomized, double-blind, placebo-controlled trial. J Allergy Clin Immunol 119: 192-198, 2007.

10. Crout DH and Vic G: Glycosidases and glycosyl transferases in glycoside and oligosaccharide synthesis. Curr Opin Chem Biol 2: 98-111, 1998.

11. Onishi N, Kawamoto S, Suzuki H, et al: Dietary pulverized konjac glucomannan suppresses scratching behavior and skin inflammatory immune responses in $\mathrm{NC} / \mathrm{Nga}$ mice. Int Arch Allergy Immunol 144: 95-104, 2007.

12. Matsuda H, Watanabe N, Geba G, et al: Development of atopic dermatitis-like skin lesion with IgE hyperproduction in $\mathrm{NC} / \mathrm{Nga}$ mice. Int Immunol 9: 461-466, 1997.

13. Tanabe S, Kinuta Y and Saito Y: Bifidobacterium infantis suppresses proinflammatory interleukin-17 production in murine splenocytes and dextran sodium sulfate-induced intestional inflammation. Int J Mol Med 22: 181-185, 2008.

14. Iguchi T, Kawata A, Watanabe T, Mazumder TK and Tanabe S: Fermented barley extract suppresses the development of atopic dermatitis-like skin lesions in NC/Nga mice, probably by inhibiting inflammatory cytokines. Biosci Biotechnol Biochem 73: 489-493, 2009.

15. Yano S, Umeda D, Yamashita S, et al: Dietary apigenin attenuates the development of atopic dermatitis-like skin lesions in $\mathrm{NC} /$ Nga mice. J Nutr Biochem (In press).

16. Leung DY: Atopic dermatitis: new insights and opportunities for therapeutic intervention. J Allergy Clin Immunol 105: 860$876,2000$.

17. Sonoyama K, Watanabe H, Watanabe J, et al: Allergic airway eosinophilia is suppressed in ovalbumin-sensitized Brown Norway rats fed raffinose and alpha-linked galactooligosaccharide. J Nutr 135: 538-543, 2005.

18. Watanabe J, Sasajima N, Aramaki A, et al: Consumption of fructo-oligosaccharide reduces 2,4-dinitrofluorobenzene-induced contact hypersensitivity in mice. Br J Nutr 100: 339-346, 2008. 
19. Tomita K, Nagura T, Okuhara Y, et al: Dietary melibiose regulates Th cell response and enhances the induction of oral tolerance. Biosci Biotechnol Biochem 71: 2774-2780, 2007.

20. Bettelli E, Oukka M and Kuchroo VK: TH-17 cells in the circle of immunity and autoimmunity. Nat Immunol 8: 345-350, 2007.

21. Steinman L: A brief history of TH17, the first major revision in the TH1/TH2 hypothesis of T cell-mediated tissue damage. Nat Med 13: 139-145, 2007.

22. Koga C, Kabashima K, Shiraishi N, et al: Possible pathogenic role of Th17 cells for atopic dermatitis. J Invest Dermatol 128 2625-2630, 2008.

23. Vulevic J, Drakoularakou A, Yaqoob P, et al: Modulation of the fecal microflora profile and immune function by a novel transgalactooligosaccharide mixture (B-GOS) in healthy elderly volunteers. Am J Clin Nutr 885: 1438-1446, 2008.
24. Ito R, Kita M, Shin-Ya M, et al: Involvement of IL-17A in the pathogenesis of DSS-induced colitis in mice. Biochem Biophys Res Commun 377: 12-16, 2008.

25. Wu S, Rhee KJ, Albesiano E, et al: A human colonic commensal promotes colon tumorigenesis via activation of T helper type 17 T cell responses. Nat Med (In press).

26. Kukkonen K, Kuitunen M, Haahtela T, et al: High intestinal IgA associates with reduced risk of IgE-associated allergic diseases. Pediatr Allergy Immunol (In press).

27. Sato T, Nakamura Y and Ozawa O: Effects of dietary galacooligosaccharides on immune system in mice. J Jpn Soc Nutr Food Sci. 61: 79-88, 2007 (in Japanese). 